\title{
Measuring Strain Fields surrounding Grain-Boundary Dislocations in Silicon using Scanning Transmission Electron Microscopy
}

\author{
Martin Couillard ${ }^{1}$ \\ 1. National Research Council Canada, Energy, Mining and Environment Portfolio, 1200 Montreal rd., \\ Ottawa ON, K1A0R6, Canada
}

Mapping atomic displacement at the nanoscale is crucial for developing and optimizing strain-engineered devices. In today's transistors, a slight deformation of the silicon lattice in the source-drain regions enhances their performance. And in the future, strain-induced electro-optic effects may potentially form the basis of an all-silicon electronic and photonic device. Transmission electron microscopy (TEM) has been established as a reliable tool to quantitatively measure atomic displacements at high spatial resolution. Several approaches have proven to be successful, and include techniques based on diffraction and holography. Alternatively, strain fields can also be extracted directly from atomic resolution images with peak finding, or geometric phase analysis (GPA) [1]. A major challenge in applying these methods to conventional high-resolution TEM has been to identify errors that may propagate through data processing. TEM imaging is particularly sensitive to the defocus value and the specimen thickness, thus, the observed variations of lattice fringes may not necessarily correspond to real atomic displacement.

Here, we perform strain analysis on annular dark-field (ADF) images acquired in an aberration- corrected scanning transmission electron microscope (STEM). ADF-STEM provides contrast dependent on the atomic number $(Z)$ and peaked at the atomic columns, rendering the interpretation of images more intuitive [2]. Chemical information can also be extracted from Z-contrast images, often with single-atom sensitivity [3], and the atomic structures of heterointerfaces can be directly interpreted [4]. Figure 1(a) presents an ADF image of dislocations located at a $\Sigma 9(122)$ grain boundary in silicon. The bottom inset displays a close-up of two dislocations, and the top inset displays the diffractogram of the image. The images were acquired with a CEOS-corrected FEI $\operatorname{Titan}^{3}$ 80-300 at the National Research Council Canada.

Because STEM relies on a serial acquisition, as opposed to a parallel acquisition as in TEM, the precision of strain maps will be limited by scanning effects. We have previously identified two major categories [5]. The first is the flyback error, which consists of a beam shift between scan lines. On the diffractogram, the flyback error introduces vertical streaks (inset of figure 1(a)), which results in strain maps that are more accurate along the scan direction. A comparison of $\varepsilon_{\mathrm{xx}}$ and $\varepsilon_{\mathrm{yy}}$ maps obtained from GPA, in figure 1(b) and (c) respectively, clearly highlights this effect. By changing the scan direction, the accuracy of $\varepsilon_{\mathrm{yy}}$ maps is improved, but tensor elements such as $\varepsilon_{x y}$ and $\omega_{x y}$ remain strongly affected. Furthermore the strain analysis from one image is limited to a single direction. The second category includes other instabilities arising from electromagnetic fields, sample drift, vibrations or other environmental noise. Since acquisition times in STEM are typically higher that in TEM, these instabilities have more impact. To reduce the scanning effects, we analyze a series of multiple rapid STEM acquisitions, and demonstrate an order of magnitude improvement in the measurements of all strain field tensor components, allowing for a direct comparison with the elastic theory. As can be seen in figure 2, the analysis results in the simultaneous determination of both the dislocation structure, with all the atomic columns visible, and the strain fields surrounding these dislocations. 


\section{References:}

[1] M.J. Hytch, E. Snoeck and R. Kilaas, Ultramicroscopy 74 (1998), p. 131

[2] D.A. Muller, Nature Materials 8 (2009), p. 263

[3] M. Couillard, G. Radtke, A.P. Knights and G.A. Botton, Phys. Rev. Lett. 107 (2011), 186104.

[4] G. Radtke, M. Couillard, G.A. Botton, D. Zhu and C.J. Humphreys, Appl. Phys. Lett. 100 (2012), 011910

[5] M. Couillard, G. Radtke and G.A. Botton, Phil. Mag. 93 (2013), p. 1250
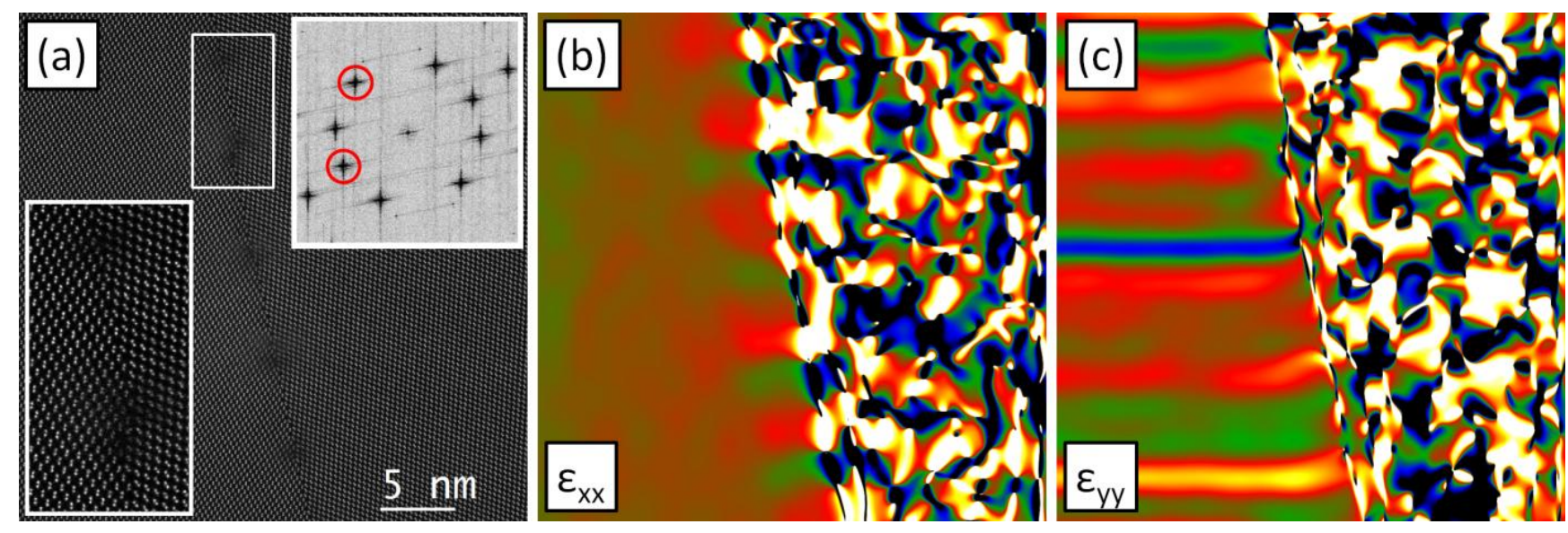

Figure 1. (a) ADF-STEM image of a $\Sigma 9(122)$ grain boundary in silicon with multiple dislocations. Insets display a close-up of dislocations and a diffractogram of the image. (b) and (c) are $\varepsilon_{\mathrm{xx}}$ and $\varepsilon_{\mathrm{yy}}$ maps extracted from a geometric phase analysis of the image using the reflections corresponding to the left grain (see diffractogram inset of (a)).
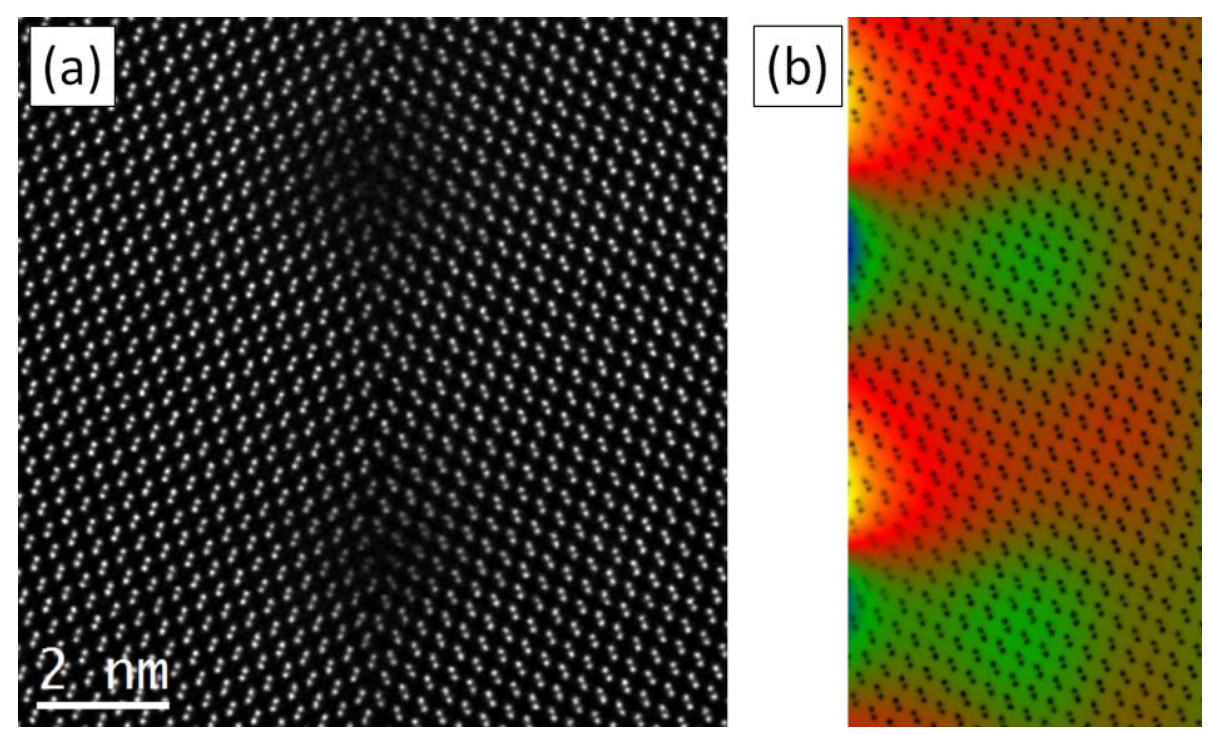

Figure 2. (a) ADF-STEM image of two edge dislocations trapped at the grain boundary. (b) Overlap for the right grain of the $\varepsilon_{\mathrm{xx}}$ map and the atomic structure. 